\title{
Urinary Excretion of Melatonin and Association with Breast Cancer: Meta-Analysis and Review of the Literature
}

\author{
Michelle Basler ${ }^{a, b} \quad$ Alexander Jetter ${ }^{a} \quad$ Daniel Fink $^{b} \quad$ Burkhardt Seifert ${ }^{c}$ \\ Gerd A. Kullak-Ublick ${ }^{a}$ Andreas Trojan ${ }^{a, d}$ \\ ${ }^{a}$ Department of Clinical Pharmacology and Toxicology, \\ ${ }^{b}$ Department of Gynecology, University Hospital Zurich, \\ ${ }^{c}$ Division of Biostatistics, Institute of Social and Preventive Medicine, University of Zurich, \\ ${ }^{\mathrm{d} B r e a s t-C e n t e r ~ Z u ̈ r i c h ~ a n d ~ O n k o z e n t r u m ~ K l i n i k ~ i m ~ P a r k, ~ Z u r i c h, ~ S w i t z e r l a n d ~}$
}

\section{Keywords}

Melatonin · aMT6s · Urinary excretion - Meta-analysis . Breast cancer

\section{Summary}

Background: Melatonin is an endocrine hormone secreted by the pineal gland during night hours that provides several biological functions in the circadian rhythm of humans. Due to anti-estrogenic properties, melatonin is considered to exhibit a protective role against the development of breast cancer (BC). Moreover, disruption of melatonin production through environmental influences, such as night work, is assumed to be a risk factor for BC. Materials and Methods: We reviewed recent findings concerning biological effects of melatonin on BC and conducted a meta-analysis to evaluate the association between melatonin and BC incidence. In random and fixed effects statistical models, concentrations (tertiles, quartiles) of the primary urinary metabolite of melatonin, 6-sulfatoxymelatonin (aMT6s), were tested for the assumption that women with the highest values would exhibit a lower risk of BC. Results: Statistical analysis of data from 5 prospective case-control studies indicates an inverse association between $\mathrm{BC}$ risk and the highest levels of urinary aMT6s. This effect seems to be influenced by lag intervals between aMT6s collection and the occurrence of $\mathrm{BC}$, timing and methods of urine sampling, as well as genetic and environmental factors. Conclusion: On the basis of the results of our meta-analysis, melatonin is likely to affect BC occurrence in women. However, methodological dissonances may require further studies.

\section{Introduction}

Melatonin (N-acetyl-5-methoxytryptamine), an endocrine hormone that is secreted primarily by the pineal gland during night hours, provides several biological functions in the circadian rhythm of humans. In 1978, Cohen et al. [1] proposed the hypothesis that a reduced function of the pineal gland and thus a concomitant decrease in melatonin levels might promote the development of breast cancer (BC) in humans. Later, Tamarkin et al. [2] and Bartsch et al. [3] found an inverse relationship between nocturnal plasma melatonin peak concentrations in women, according to Tamarkin et al. [2] particularly in those with estrogen receptor (ER)-positive BC. Several studies also described lower rates of BC occurrence in blind women, thereby indicating a causative relationship between lower $\mathrm{BC}$ rates and an unaffected melatonin secretion [4-6]. Meanwhile, melatonin was demonstrated to modulate the estrogen-signaling pathway in hormone-dependent mammary tumors and to downregulate gonadal estrogen synthesis [7]. Melatonin acts as a selective estrogen receptor modulator (SERM) and prevents estrogen-induced effects by disrupting the binding of the estrogen-ER $\alpha$-calmodulin complex to the ER-binding element on DNA, thereby inhibiting estrogenrelated transcription [8, 9]. Physiological concentrations of melatonin also decrease aromatase expression in MCF-7 human BC cell lines, and promote a synergistic anti-proliferative effect with tamoxifen in anti-tumoral endocrine therapy $[10,11]$. Probably unaffected by expression of melatonin MT1 receptors on $\mathrm{BC}$ cells, administration of melatonin at physiological concentrations also abrogates extragonadal steroid production via aromatases $[12,13]$. Several animal models demonstrated that melatonin administration significantly reduces the occurrence of mammary tumors [10], in relation to which a physiological circadian synchronization seems to be

\section{KARGER \\ Fax +497614520714 \\ Information@Karger.com}

www.karger.com (c) 2014 S. Karger GmbH, Freiburg

1661-3791/14/0093-0182\$39.50/0

Accessible online at:

www.karger.com/brc
PD Dr. med. Andreas Trojan

Breast-Center Zürich and

OnkoZentrum Klinik im Park

Seestrasse 214, 8008 Zürich, Switzerland

andtrojan@yahoo.de 
superior to single daily exogenous melatonin application in terms of disease progression $[14,15]$. Melatonin was also found to protect animals from adriamycin-induced cardiotoxicity, while co-administration of both substances seems to enhance cytotoxic effects, thereby allowing for dose reductions and attenuation of undesirable side effects [16]. However, a variety of receptor-independent anti-carcinogenic effects of melatonin, such as free radical scavenging via antioxidant properties and modulation of immune functions [17], might exist as well that render the drug potentially useful in the treatment of other diseases.

In 2007, the WHO International Agency for Research on Cancer (IARC) classified 'shift work that involves circadian disruption as probably carcinogenic to humans' [18]. While several studies on mammalian animal models confirmed carcinogenicity of light during the biological night, evidence in humans appears fragmentary [19, 20]. However, an increased risk for BC has been observed among women who worked periods of rotating night shifts [21], although this effect seems to be related to a high quantity of night shifts and the cumulative number of years ( $>6$ years) a person has worked nights, and in particular depends on whether a night worker is a morning chronotype [22]. In contrast, a recent study [23] associated an increasing incidence of $\mathrm{BC}$ with small numbers of night shifts per week, indicating a potential relationship to more frequent disruptions of the circadian rhythm with deranged adaption to changes between day and night schedules [24]. In young women, a night shift-associated BC risk might even exist before the first full-term pregnancy since the mammary gland is not fully differentiated until first childbirth and lactation [25].

Whether relative concentrations or the absolute decrease of melatonin levels with increasing years of sleep disturbance as well as sleep duration might affect the risk of $\mathrm{BC}$ remains inconclusive [26-31], since many studies do not provide quantitative urinary or serum melatonin concentrations. Independent of melatonin dynamics, interpretation of studies might be complicated by the expression of circadian clock genes, such as cell cycle regulators p53 and c-myc, which directly affect apoptosis and proliferation, as well as potential differences in culture, diet, immune reactivity, and genetic variations [32,
33]. Here we provide an overview of the recent findings concerning the biology and impact of melatonin in relation to $\mathrm{BC}$ occurrence. A meta-analysis was conducted to quantitatively assess a possible association between melatonin and $\mathrm{BC}$ incidence based on studies of urinary melatonin concentrations.

\section{Materials and Methods}

Melatonin is metabolized in the liver and exhibits a short half-life of about $40 \mathrm{~min}$ [34]. The main degradation pathway consists of 6-hydroxylation followed by conjugation for renal elimination. The concentration of the primary urinary metabolite of melatonin, 6-sulfatoxymelatonin (aMT6s) reflects the amount of nocturnal plasma melatonin in the body [35]. Quantitative assessment of aMT6s in urine is deemed a practical method to determine the circadian phase since it represents the amount of endogenous melatonin during collection time and is thus considered a reliable approximation of melatonin production in an individual, even if urine is stored over prolonged periods of time [36]. Although small phase changes could be monitored by frequent sampling of blood or saliva [37], simple morning urine aMT6s measurement does not interfere with the participant's sleeping pattern. Despite a large variability in melatonin amplitudes, their range remains relatively stable within the same person [38], justifying singular melatonin measurements as is conducted in most recent studies. For our meta-analysis based on urinary melatonin concentrations, 2 sampling techniques were applied: first morning urine and 24-h urine collection. Studies that focused on aspects such as duration of sleep and tumor size were excluded in the numerical analysis. The data source for the meta-analysis was provided by the electronic database PubMed, and our review comprises only English articles from 1989 to 2013. Search terms included melatonin, breast cancer, and urinary sulfatoxymelatonin (aMT6s).

\section{Statistical Considerations and Results}

A total of 24 search results were found of which 10 studies measured the urinary aMT6s concentration as a surrogate of circulating melatonin levels: Skene et al. (1990) [39], Bartsch et al. (1997) [40], Travis et al. (2004) [41], Schernhammer et al. (2005) [42], Wu et al. (2008) [31], Schernhammer et al. (2008) [43], Schernhammer et al. (2009) [44], Schernhammer et al. (2010) [45], Davis et al. (2012) [46], and Wu et al. (2013) [47]. Of these 10 studies, 5 displayed their data in a similar manner and met the criteria of this meta-analysis (table 1).

Table 1. Overview of the studies used for the present meta-analysis

\begin{tabular}{|c|c|c|c|c|c|}
\hline First author [ref.] & Year & Data & Urine sample & Menopausal status & Country or cohort and time period of enrollment \\
\hline Travis [41] & 2004 & tertiles & 24-h urine & $\begin{array}{l}\text { pre- and } \\
\text { postmenopausal }\end{array}$ & $\begin{array}{l}\text { Island of Guernsey (British crown dependency), Guernsey III } \\
\text { Study; 1977-1985 }\end{array}$ \\
\hline Schernhammer [42] & 2005 & quartiles & $\begin{array}{l}\text { first morning } \\
\text { urine }\end{array}$ & $\begin{array}{l}\text { primarily } \\
\text { premenopausal }\end{array}$ & $\begin{array}{l}\text { USA, Nurses' Health Study II (NHSII) cohort (1989); 1996-1999 } \\
\text { (urine collection) }\end{array}$ \\
\hline Schernhammer [43] & 2008 & quartiles & $\begin{array}{l}\text { 12-h overnight } \\
\text { urine }\end{array}$ & postmenopausal & $\begin{array}{l}\text { Italy, Hormones and Diet in the Etiology of Breast Cancer Risk } \\
\text { cohort (ORDET cohort, postmenopausal); 1987-1992 }\end{array}$ \\
\hline Schernhammer [44] & 2009 & quartiles & $\begin{array}{l}\text { first morning } \\
\text { urine }\end{array}$ & postmenopausal & $\begin{array}{l}\text { USA, Nurses' Health Study (NHS) cohort (1976); 2000-2002 } \\
\text { (urine collection) }\end{array}$ \\
\hline Schernhammer [45] & 2010 & quartiles & $\begin{array}{l}\text { 12-h overnight } \\
\text { urine }\end{array}$ & premenopausal & $\begin{array}{l}\text { Italy, Hormones and Diet in the Etiology of Breast Cancer Risk } \\
\text { cohort (ORDET cohort, premenopausal); 1987-1992 }\end{array}$ \\
\hline
\end{tabular}


Table 2. Data used for urinary melatonin meta-analysis (Event $1=$ cases in the highest quartile, Event $2=$ cases in the lowest quartile, $\mathrm{n}=$ total of respective quartile; case subjects defined as women who developed breast cancer after their enrollment in the study cohort; matched control subjects were randomly chosen, alive, and free of cancer at the time of diagnosis of the index case subject)

\begin{tabular}{lllrrll}
\hline First author [ref.] & Year & Event 1 & n 1 & Event 2 & n 2 & Comment \\
\hline Travis [41] & 2004 & 46 & 163 & 39 & 158 & tertiles, 24-h urine, pre- and postmenopausal \\
Schernhammer [42] & 2005 & 23 & 96 & 50 & 123 & first morning urine, primarily premenopausal \\
Schernhammer [43] & 2008 & 40 & 218 & 56 & 233 & 12-h overnight urine, postmenopausal \\
Schernhammer [44] & 2009 & 75 & 210 & 107 & 243 & first spot morning urine, postmenopausal \\
Schernhammer [45] & 2010 & 30 & 113 & 28 & 93 & 12-h overnight urine, premenopausal \\
\hline
\end{tabular}

Fig. 1. Forest plot illustrating the combined estimated risk ratio for breast cancer from the meta-analysis.

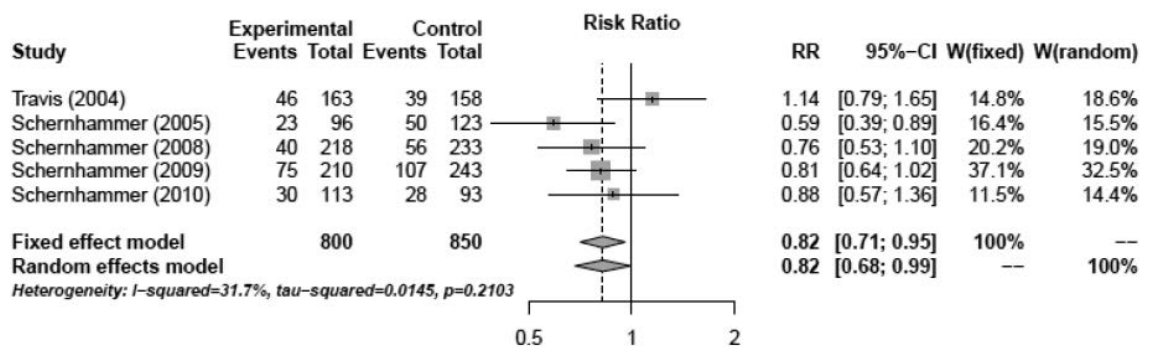

To evaluate a potential publication bias, a funnel plot was constructed. The linear regression test of funnel plot asymmetry was performed and suggests that the data is distributed evenly and there is no indication of a selection bias $(\mathrm{p}=0.94)$. Although the number of studies seems too small to exclude selection bias, the $\mathrm{p}$ value and funnel plot (figures not shown) do not indicate evidence thereof.

Conclusions drawn from these 5 evaluable studies can be summarized in brief as follows: Travis et al. [41] found no significant difference in urinary melatonin concentrations in BC patients and controls (Guernsey III study). In 2005 (NHSII) and 2008 (ORDET cohort), Schernhammer et al. $[42,43]$ reported that increased melatonin levels were statistically significantly associated with lower risk of invasive $\mathrm{BC}$ in pre- and postmenopausal women. This inverse association was again confirmed by Schernhammer et al. in 2009 (NHS) [44], while in 2010, Schernhammer et al. also reported a positive association between aMT6s concentration and BC risk in premenopausal women (ORDET cohort) [45]. In particular, when cases were excluded which exhibited a lag time below 2 years between urine collection and $\mathrm{BC}$ diagnosis, melatonin had a protective effect on premenopausal BC, i.e. an inverse association could be observed [45]. Since most samples from the recent study by $\mathrm{Wu}$ et al. [47] were not derived from first morning voids but from randomly timed spot urine, unfortunately, this study was deemed not suitable for our meta-analysis.

Upon statistical considerations concerning these studies, melatonin concentrations were classified into quartiles or tertiles according to the one-time overnight urinary aMT6s concentration (table 2). Since our assumption was that women in the highest quartile (exhibiting the highest melatonin values) would experience a lower risk of developing BC compared to women in the lowest quartile (i.e. exhibiting a risk ratio smaller than 1 ), the relative $\mathrm{BC}$ risks of the highest and lowest quartile (tertile respectively) were determined, compared, and used to calculate the corresponding odds ratios.

An important factor, which was taken into account, is the lag time between urine sample collection and diagnosis of BC. Since the presence of a preclinical tumor might have influenced the basic melatonin level, 2 studies [41, 45] excluded individuals in which cancer diagnosis occurred within less than 2 years of urine collection (data not shown). However, Travis et al. [41] reported unchanged results when an adjustment for a 2-year lag time was performed. In the 2008 study by Schernhammer et al. [43], the mean time between urine collection and diagnosis was 80 months $( \pm 50$ months standard deviation (SD)), while in Schernhammer et al. 2009 [44] this time interval was only 30 months ( \pm 18 months SD).

Using the data with a 2-year lag time (where specified), led to the following considerations: To decide whether to use a fixed or random effects model in this meta-analysis, a test of heterogeneity (Cochran's Q) was performed and produced a $\mathrm{p}$ value of 0.21 . In meta-analyses with a fixed effect, it is assumed that the same effect underlies each study and that the observed variability occurs only due to sample variability. However, with a p value (obtained from the test of homogeneity) higher than 0.05 , the null hypothesis cannot be rejected, i.e. this $\mathrm{p}$ value indicates no evidence against homogeneity. Consequently, the fixed effects model could be applied. As mentioned above, the sample size is considered small, and hence the Cochran's Q test has low power. In addition to sample variability, however, analysis may also be performed on the assumption that the underlying effect between the studies varies randomly [48]. Using the random and fixed effects models, a relative risk of 0.82 was obtained. Statistical significance is attained in the random as well as in the fixed effects model: $95 \%$ confidence interval $(0.68 ; 0.99)$, p value 0.04 for the random effects model, and $95 \%$ confidence interval $(0.71$; $0.95)$, $\mathrm{p}$ value 0.01 for the fixed effects model. Although not 
all included studies suggest an effect in the same direction, the confidence intervals are overlapping in all of them. The combined estimated risk ratio is smaller than 1 and hence implies that a reduced $\mathrm{BC}$ risk prevails in women with higher melatonin levels (fig. 1).

\section{Discussion}

We identified 5 prospective case-control studies that met the inclusion criteria and were finally used for statistical calculations. Although Schernhammer is co-author in 4 of the 5 studies, their data sets can be considered different from each other (table 1, funnel plot). The analysis also revealed the importance of a defined lag time between urine collection and $\mathrm{BC}$ diagnosis in order to appropriately assess combined risk evaluations for $\mathrm{BC}$ upon urinary melatonin excretion [45]. In this regard, the ORDET cohort in premenopausal women found highly variable odds ratios, while a significant $\mathrm{BC}$ risk reduction of $34 \%$ in the highest category of aMT6s levels (combined estimate 0.66 ) was obtained only when data with a lag time of $>2$ years were analyzed [41, 45, 47]. In contrast, adjustment for a 2-year lag time presented unchanged results in the study by Travis et al. [41]. However, the studies by Travis et al. [41] and $\mathrm{Wu}$ et al. [47] potentially failed to demonstrate a protective effect of melatonin due to the variable timing of sample collection; both studies were based on measurement of non-timed urine collections (24-h and randomly timed spot urine). Considering the importance of lag-time, our meta-analysis apparently displays a trend towards a protective effect of melatonin on BC occurrence, although only marginally significant (fig. 1).

Biological effects of melatonin might also be compromised by mutations in the melatonin receptors (MTNR1a and MTNR1b) as well as alterations of the enzyme arylalkylamine $\mathrm{N}$-acetyltransferase (AA-NAT) which catalyzes a key step of melatonin synthesis in the pineal gland, and gets rapidly inactivated when exposed to light at night $[49,50]$. Whilst a genome-wide association study from the Shanghai Breast Cancer Study discovered single nucleotide polymorphisms concerning the melatonin receptor expression, an association with variable susceptibility to $\mathrm{BC}$ may correspond to different phenotypes within a population of the same racial origin [51]. Consequently, results and dynamics of melatonin concentration from Chinese cohorts [47] need to be interpreted with respect to epigenetic modifications, menopausal status, and dietary as well as environmental variables such as sleep deprivation and compromised immune function [52-54].

While most studies and our meta-analysis evaluated melatonin levels before $\mathrm{BC}$ occurrence, assessments of melatonin levels in patients with manifest cancer indicate that the tumors themselves might affect melatonin secretion upon systemic endocrine reactivity towards cancer. Higher melatonin levels seemed prevalent in untreated BC patients and those with a favorable prognosis, i.e. ER-positive and node-negative tumors [55-57], although this trend was no longer seen in older and postmenopausal women in whom circulating aMT6s levels seem to decline [39]. In contrast, low aMT6s excretion was reported with increasing tumor size, and the melatonin amplitude normalized again after tumor removal [3, 40].

So far, available studies provide only little information on how to determine which collection time would optimally reflect biological effects of melatonin. Although urinary aMT6s is deemed an appropriate metabolite associated with non-invasive collection procedures, it only represents a surrogate for endogenous periodical melatonin secretion. Since studies measured aMT6s concentrations e.g. over $24 \mathrm{~h}, 12 \mathrm{~h}$, or in the first morning urine as well as randomly timed spot urine, that information cannot reliably be combined for calculations. Moreover, 24-h urinary melatonin assessment may reflect the nocturnal increase in melatonin concentrations, but does not consider peak and minimum values. Wu et al. [47] therefore suggested that randomly timed spot urine samples are not suitable as a surrogate for melatonin concentrations in humans and even provide an inaccurate comparison for women in the same cohort. Since it remains unclear to what extent a preclinical breast tumor might influence melatonin concentrations, supplemental information on lag times seems mandatory $[39,41,47]$.

Thus, further investigations need to evaluate methods of melatonin assessment in order to facilitate the comparison of different studies and ethnic groups with a focus on longitudinal analysis and dynamics of melatonin secretion in individuals before tumor discovery and under treatment. Finally, clinical trials designed to exogenously administer melatonin may be able to confirm effects of melatonin on BC incidence and provide information on dosage and long-term safety. For this, appropriate (range between the 75th and 100th percentile) levels of urinary aMT6s could be achieved e.g. by administration of synthetic melatonergic agonists with extended halflives [34, 58]. Alternatively, filter glasses and light systems that block blue spectra and short-term exposure to bright artificial light should protect shift workers from an unintended melatonin decline [20,59-61]. In the light of the present trend towards a 24-h society, the current data on the effects of melatonin may warrant further investigations with a focus also on cancer prevention in men [62].

\section{Acknowledgement}

This work was supported by the Swiss Tumor Institute, Zurich, Switzerland.

\section{Disclosure Statement}

All authors and coauthors indicated no potential conflicts of interest. 


\section{References}

1 Cohen M, Lippman M, Chabner B: Role of pineal gland in aetiology and treatment of breast cancer. Lancet 1978;2:814-816.

2 Tamarkin L, Danforth D, Lichter A, et al.: Decreased nocturnal plasma melatonin peak in patients with estrogen receptor positive breast cancer. Science 1982;216:1003-1005.

3 Bartsch C, Bartsch H, Fuchs U, Lippert TH, Bellmann O, Gupta D: Stage-dependent depression of melatonin in patients with primary breast cancer. Correlation with prolactin, thyroid stimulating hormone, and steroid receptors. Cancer 1989;64:426-433.

4 Feychting M, Osterlund B, Ahlbom A: Reduced cancer incidence among the blind. Epidemiology 1998;9:490-494.

5 Kliukiene J, Tynes T, Andersen A: Risk of breast cancer among Norwegian women with visual impairment. Br J Cancer 2001;84:397-399.

6 Flynn-Evans EE, Stevens RG, Tabandeh H, Schernhammer ES, Lockley SW: Total visual blindness is protective against breast cancer. Cancer Causes Control 2009;20:1753-1756.

7 Sánchez-Barceló EJ, Cos S, Mediavilla D, MartínezCampa C, González A, Alonso-González C: Melatonin-estrogen interactions in breast cancer. J Pineal Res 2005;38:217-222.

8 Rato AG, Pedrero JG, Martinez MA, del Rio B, Lazo PS, Ramos S: Melatonin blocks the activation of estrogen receptor for DNA binding. FASEB J 1999;13:857-868.

$\checkmark 9$ Del Río B, García Pedrero JM, Martínez-Campa C, Zuazua P, Lazo PS, Ramos S: Melatonin, an endogenous-specific inhibitor of estrogen receptor alpha via calmodulin. J Biol Chem 2004;279:38294 38302 .

\10 Cos S, González A, Güezmes A, et al.: Melatonin inhibits the growth of DMBA-induced mammary tumors by decreasing the local biosynthesis of estrogens through the modulation of aromatase activity. Int J Cancer 2006;118:274-278.

11 Wilson ST, Blask DE, Lemus-Wilson AM: Melatonin augments the sensitivity of MCF-7 human breast cancer cells to tamoxifen in vitro. J Clin Endocrinol Metab 1992;75:669-670.

12 Ram PT, Dai J, Yuan L, et al.: Involvement of the $\mathrm{mt} 1$ melatonin receptor in human breast cancer Cancer Lett 2002;179:141-150.

13 Russo IH, Russo J: Role of hormones in mammary cancer initiation and progression. J Mammary Gland Biol Neoplasia 1998:3:49-61.

14 Tamarkin L, Cohen M, Roselle D, Reichert C, Lippman M, Chabner B: Melatonin inhibition and pinealectomy enhancement of 7,12-dimethylbenz(a)anthracene-induced mammary tumors in the rat. Cancer Res 1981;41:4432-4436.

$\checkmark 15$ Hill SM, Cheng C, Yuan L, et al.: Age-related decline in melatonin and its MT1 receptor are associated with decreased sensitivity to melatonin and enhanced mammary tumor growth. Curr Aging Sci 2013;6:125-133.

16 Kim C, Kim N, Joo H, et al.: Modulation by melatonin of the cardiotoxic and antitumor activities of adriamycin. J Cardiovasc Pharmacol 2005;46:200210.

17 Grant SG, Melan MA, Latimer JJ, Witt-Enderby PA: Melatonin and breast cancer: cellular mechanisms, clinical studies and future perspectives. Expert Rev Mol Med 2009;11:e5.

18 Straif K, Baan R, Grosse Y, et al.: Carcinogenicity of shift-work, painting, and fire-fighting. Lancet Oncol 2007;8:1065-1066.
Reiter RJ, Tan DX, Korkmaz A, Erren TC, Piekarski C, Tamura H, Manchester LC: Light at night, chronodisruption, melatonin suppression, and cancer risk: a review. Crit Rev Oncog 2007; 13:303-328.

20 Stevens RG, Blask DE, Brainard GC, et al.: Meeting report: the role of environmental lighting and circadian disruption in cancer and other diseases. Environ Health Perspect 2007;115:1357-1362.

21 Davis S, Mirick DK, Stevens RG: Night shift work, light at night, and risk of breast cancer. J Natl Cancer Inst 2001;93:1557-1562.

22 Hansen J, Lassen CF: Nested case-control study of night shift work and breast cancer risk among women in the Danish military. Occup Environ Med 2012;69:551-556.

23 Menegaux F, Truong T, Anger A, et al.: Night work and breast cancer: a population-based casecontrol study in France (the CECILE study). Int J Cancer 2013;132:924-931.

24 Stevens RG, Hansen J, Costa G, et al.: Considerations of circadian impact for defining 'shift work' in cancer studies: IARC Working Group Report. Occup Environ Med 2011;68:154-162.

25 Trichopoulos D, Adami HO, Ekbom A, Hsieh CC, Lagiou P: Early life events and conditions and breast cancer risk: from epidemiology to etiology. Int J Cancer 2008;122:481-485.

26 Pinheiro SP, Schernhammer ES, Tworoger SS, Michels KB: A prospective study on habitual duration of sleep and incidence of breast cancer in a large cohort of women. Cancer Res 2006;66:55215525.

27 Verkasalo PK, Lillberg K, Stevens RG, et al.: Sleep duration and breast cancer: a prospective cohort study. Cancer Res 2005;65:9595-9600.

28 Patel SR, Ayas NT, Malhotra MR, et al.: A prospective study of sleep duration and mortality risk in women. Sleep 2004;27:440-444.

29 Tamakoshi A, Ohno Y, Group JS: Self-reported sleep duration as a predictor of all-cause mortality: results from the JACC study, Japan. Sleep 2004; 27:51-54.

30 Kakizaki M, Kuriyama S, Sone T, et al.: Sleep duration and the risk of breast cancer: the Ohsaki Cohort Study. Br J Cancer 2008;99:1502-1505.

31 Wu AH, Wang R, Koh WP, Stanczyk FZ, Lee HP, Yu MC: Sleep duration, melatonin and breast cancer among Chinese women in Singapore. Carcinogenesis 2008;29:1244-1248.

32 Costa G, Haus E, Stevens R: Shift work and cancer - considerations on rationale, mechanisms, and epidemiology. Scand J Work Environ Health 2010;36:163-179.

33 Pronk A, Ji BT, Shu XO, et al.: Night-shift work and breast cancer risk in a cohort of Chinese women. Am J Epidemiol 2010;171:953-959.

34 Aeschbach D, Lockyer BJ, Dijk DJ, Lockley SW, Nuwayser ES, Nichols LD, Czeisler CA: Use of transdermal melatonin delivery to improve sleep maintenance during daytime. Clin Pharmacol Ther 2009;86:378-382.

35 Graham C, Cook MR, Kavet R, Sastre A, Smith DK: Prediction of nocturnal plasma melatonin from morning urinary measures. J Pineal Res 1998; 24:230-238.

36 Travis RC, Allen NE, Peeters PH, van Noord PA, Key TJ: Reproducibility over 5 years of measurements of 6-sulphatoxymelatonin in urine samples from postmenopausal women. Cancer Epidemiol Biomarkers Prev 2003;12:806-808.
Benloucif S, Burgess HJ, Klerman EB, et al. Measuring melatonin in humans. J Clin Sleep Med 2008;4:66-69.

38 Arendt J: Melatonin and the Mammalian Pineal Gland. London, Chapman \& Hall, 1995.

39 Skene DJ, Bojkowski CJ, Currie JE, Wright J, Boulter PS, Arendt J: 6-sulphatoxymelatonin production in breast cancer patients. J Pineal Res 1990;8:269-276.

40 Bartsch C, Bartsch H, Karenovics A, Franz H, Peiker G, Mecke D: Nocturnal urinary 6-sulphatoxymelatonin excretion is decreased in primary breast cancer patients compared to age-matched controls and shows negative correlation with tumor-size. J Pineal Res 1997;23:53-58.

41 Travis RC, Allen DS, Fentiman IS, Key TJ: Melatonin and breast cancer: a prospective study. J Natl Cancer Inst 2004;96:475-482.

42 Schernhammer ES, Hankinson SE: Urinary melatonin levels and breast cancer risk. J Natl Cancer Inst 2005;97:1084-1087.

43 Schernhammer ES, Berrino F, Krogh V, et al.: Urinary 6-sulfatoxymelatonin levels and risk of breast cancer in postmenopausal women. J Natl Cancer Inst 2008;100:898-905.

44 Schernhammer ES, Hankinson SE: Urinary melatonin levels and postmenopausal breast cancer risk in the Nurses' Health Study cohort. Cancer Epidemiol Biomarkers Prev 2009;18:74-79.

45 Schernhammer ES, Berrino F, Krogh V, et al: Urinary 6-sulphatoxymelatonin levels and risk of breast cancer in premenopausal women: the ORDET cohort. Cancer Epidemiol Biomarkers Prev 2010;19:729-737.

46 Davis S, Mirick DK, Chen C, Stanczyk FZ: Night shift work and hormone levels in women. Cancer Epidemiol Biomarkers Prev 2012;21:609618.

$47 \mathrm{Wu}$ AH, Stanczyk FZ, Wang R, Koh WP, Yuan JM, Yu MC: Sleep duration, spot urinary 6-sulfatoxymelatonin levels and risk of breast cancer among Chinese women in Singapore. Int J Cancer 2013;132:891-896.

48 Held L, Rufibach K, Burkhardt S: Einführung in die Biostatistik, 4th ed. University of Zurich, 2009.

49 Coon SL, Roseboom PH, Baler R, et al.: Pineal serotonin $\mathrm{N}$-acetyltransferase: expression cloning and molecular analysis. Science 1995;270:16811683.

50 Klein DC, Coon SL, Roseboom PH, et al.: The melatonin rhythm-generating enzyme: molecular regulation of serotonin $\mathrm{N}$-acetyltransferase in the pineal gland. Recent Prog Horm Res 1997;52:307357; discussion 357-308.

51 Deming SL, Lu W, Beeghly-Fadiel A, et al.: Melatonin pathway genes and breast cancer risk among Chinese women. Breast Cancer Res Treat 2012; 132:693-699.

52 Bhatti P, Mirick DK, Davis S: Racial differences in the association between night shift work and melatonin levels among women. Am J Epidemiol 2013; 177:388-393.

53 Nagata C, Nagao Y, Shibuya C, Kashiki Y, Shimizu H: Association of vegetable intake with urinary 6-sulfatoxymelatonin level. Cancer Epidemiol Biomarkers Prev 2005;14:1333-1335.

54 McPherson K, Steel CM, Dixon JM: ABC of breast diseases. Breast cancer - epidemiology, risk factors, and genetics. BMJ 2000;321:624-628.

55 Barni S, Lissoni P, Sormani A, et al.: The pineal gland and breast cancer: serum levels of melatonin in patients with mammary tumors and their rela- 
tion to clinical characteristics. Int J Biol Markers 1989;4:157-162

56 Lissoni P, Viviani S, Bajetta E, Buzzoni R, Barreca A, Mauri R, et al.: A clinical study of the pineal gland activity in oncologic patients. Cancer 1986;57:837-842.

58 Hardeland R: Investigational melatonin receptor agonists. Expert Opin Investig Drugs 2010;19:747764.

59 Fung BK: Transducin: structure, function and role in phototransduction. Progress in Retinal Research 1987;6:151-177.

57 Touitou Y, Fevre-Montange M, Proust J, Klinger E, Nakache JP: Age- and sex-associated modification of plasma melatonin concentrations in man. Relationship to pathology, malignant or not, and autopsy findings. Acta Endocrinol (Copenh) 1985;108:135144.
60 Sanchez-Barcelo EJ, Mediavilla MD, AlonsoGonzalez C, Reiter RJ: Melatonin uses in oncology: breast cancer prevention and reduction of the side effects of chemotherapy and radiation. Expert Opin Investig Drugs 2012;21:819-831.
1 Blask DE, Brainard GC, Dauchy RT, et al.: Melatonin-depleted blood from premenopausal women exposed to light at night stimulates growth of human breast cancer xenografts in nude rats. Cancer Res 2005;65:11174-11184.

62 Sigurdardottir LG, Valdimarsdottir UA, Fall K, Rider JR, Lockley SW, Schernhammer E, et al: Circadian disruption, sleep loss, and prostate cancer risk: a systematic review of epidemiologic studies. Cancer Epidemiol Biomarkers Prev 2012; 21:1002-1011. 